\title{
Review \\ Role of nitric oxide in the gastrointestinal tract
}

\author{
Angel Lanas
}

Servicio de Aparato Digestivo, Hospital Clinico Zaragoza, CIBERehd, IACS, Universidad de Zaragoza, C/ Domingo Miral s/n, 50009 Zaragoza, Spain

Corresponding author: Angel Lanas, angel.lanas@gmail.com

Published: 17 October 2008

This article is online at http://arthritis-research.com/supplements/10/S2/S4

(c) 2008 BioMed Central Ltd
Arthritis Research \& Therapy 2008, 10(Suppl 2):S4 (doi:10.1186/ar2465)

The search for new agents with which to manage pain and to reduce the attendant adverse events associated with NSAID use has led to the development of a new class of agents termed cyclo-oxygenase (COX)-inhibiting, nitric oxide (NO)donating drugs (CINODs). CINODs are NO donors in which $\mathrm{NO}$ is coupled to an NSAID and could potentially improve the gastrointestinal safety profile of traditional NSAIDs $[6,7]$. This review discusses the known functions of NO in the gastrointestinal tract and some of the mechanisms that underlie NSAID-induced gastric damage. The potential benefits of NO-donating agents in the treatment of NSAID-induced gastric damage are reviewed, and finally the potential benefits of CINODs in the management of OA are discussed.

\section{Nitric oxide synthesis and nitric oxide synthase enzymes}

NO mediates multiple physiological functions in the gastrointestinal tract, including mucosal blood flow, maintenance of mucosal integrity, and maintenance of vascular tone. NO is synthesized by the conversion of L-arginine to equimolar amounts of L-citrulline and NO. Once NO is generated, it binds to the heme group of soluble guanylyl cyclase, which catalyzes the conversion of GTP to cGMP, leading to an intracellular increase in cGMP concentration [8]. cGMP then binds to and modifies the target domain of specific proteins, including protein kinases, ion channels, and phosphodiesterases, to elicit cellular responses. However, NO can also act in a cGMP-independent manner, in which redox derivatives of NO mediate cellular activities by posttranslational modifications or oxidation of proteins and/or lipids. NO works in the gastrointestinal tract to help maintain homeostasis and, when disrupted, can perpetuate pathologic conditions.

The oxidation of L-arginine to L-citrulline and NO is catalyzed by one of three isoforms of nitric oxide synthase (NOS)

CINOD = cyclo-oxygenase inhibiting nitric oxide donating; $\mathrm{COX}=$ cyclo-oxygenase; eNOS = endothelial nitric oxide synthase; iNOS $=$ inducible nitric oxide synthase; L-NAME = NG-nitro-L-arginine methyl ester; L-NMMA = NG-monomethyl-L-arginine; NO = nitric oxide; NOS = nitric oxide synthase; NSAID = nonsteroidal anti-inflammatory drug; OA = osteoarthritis; PPI = proton pump inhibitor; SNAP $=S$-nitroso- $N$-acetylpenicillamine; UGIB = upper gastrointestinal bleeding. 
enzymes. At the gastrointestinal level, the two constitutively expressed isoforms, namely endothelial NOS (eNOS) and neuronal NOS (nNOS), are expressed basally at the vascular endothelium and the enteric nervous system of the gastrointestinal tract, respectively [9]. The inducible isoform of NOS (iNOS) is expressed in macrophages and neutrophils, but with potent signals for induction it may also be observed in epithelial cells and neurons [10]. iNOS is a calcium-independent enzyme that is upregulated in response to inflammation and other stimuli, remains activated for longer periods of time compared with the other isoforms, and generates a sustained increase in NO [11]. A study of iNOS expression patterns in the neonatal and adolescent rat intestine revealed that iNOS activation in response to lipopolysaccharide-stimulation in the ileum varies with age [12]. Once iNOS is upregulated, the concentration of NO will increase, resulting in the production of reactive oxygen species and ultimately oxidative stress. NO itself, as well as ROS molecules such as superoxide and peroxynitrite (which results from the interaction between $\mathrm{NO}$ and superoxide anion) will all act to elicit downstream effects. The effects of NO reacting with other radicals can induce either deleterious or even beneficial consequences, depending on the conditions under which the process takes place [10].

\section{Nitric oxide in the gastrointestinal tract}

It is well known that COX-derived prostaglandins play multiple roles in maintaining the integrity of the gastrointestinal mucosa, including stimulation of mucus and bicarbonate secretion, resistance of epithelial cells to injury, inhibition of recruitment of leukocytes to the mucosa, and downregulating the release of inflammatory mediators [13]. It is now also clear that $\mathrm{NO}$ plays a key role in the maintenance of the gastrointestinal mucosa and that both $\mathrm{NO}$ and COX derived prostaglandins share similarities in various gastrointestinal functions. NO and prostaglandin mediated mechanisms of mucosal defense also exhibit a degree of cooperation. Suppression of one arm can lead to a compensatory elevation in the other $[10,12,14]$.

$\mathrm{NO}$ is responsible for helping to maintain the integrity of the gastric epithelium and the mucus barrier. NO is a vasodilator and mediates gastric blood flow. Experiments in rats revealed that the NOS inhibitor NG-monomethyl-L-arginine (L-NMMA) induced a dose-dependent increase in systemic blood pressure and a decrease in resting gastric mucosal blood flow [15]. There is evidence that the addition of exogenous NO, similar to that provided by a transdermal patch of nitroglycerin in humans, can help to protect the rat gastric mucosa from indomethacin-induced gastric damage, perhaps because the nitroglycerin patch maintains adequate mucosal blood flow and inhibits leukocyte-endothelial cell interactions [16].

Mucus contributes to gastrointestinal defense by acting as a physical barrier to damage as well as helping to protect the epithelium from damage caused by acid and pepsin $[10,17]$.
Brown and coworkers [18] demonstrated that incubation with $\mathrm{NO}$ stimulates mucus secretion in rat gastric mucosal cells in a dose-dependent manner and that this stimulation is dependent on cGMP. In vivo experiments [19] subsequently showed that administration of the $\mathrm{NO}$ donor isosorbide dinitrate to rat gastric lumen leads to a dose-dependent increase in mucus gel thickness, again demonstrating that under certain conditions NO helps to mediate mucus secretion to protect the gastric epithelium.

More recent studies have demonstrated that $\mathrm{NO}$ also protects the gastrointestinal tract by inhibiting gastric acid secretion. Intragastric application of NO donors, including FK409 and sodium nitroprusside, significantly decreased both basal secretion and secretion that was stimulated by pentagastrin and YM-14673, an analog of thyrotropin-releasing hormone [17]. In addition, incubation with $N{ }^{G}$-nitro-L-arginine methyl ester (L-NAME), a NOS inhibitor, enhanced pentagastrin and YM-14673 stimulated acid secretion but not basal secretion [20]. These experiments demonstrated that NO helps to decrease acid secretion from gastric parietal cells.

Subsequent experiments demonstrated that when isolated human gastric gland cells were incubated with the NO donor $S$-nitroso- $N$-acetylpenicillamine (SNAP), stimulation with histamine resulted in decreased acid secretion when compared with controls [21]. Because acid secretion in the presence of a cGMP analog was inhibited and the presence of SNAP and a GC synthase inhibitor did not result in a decrease in gastric acid secretion, the authors concluded that the role of $\mathrm{NO}$ in gastric acid secretion is cGMPdependent.

Although prostaglandins and $\mathrm{NO}$ are required for normal gastrointestinal function, there is also some evidence that a large excess of these compounds may have deleterious effects on the gastrointestinal tract. COX-2, the inducible isoenzyme of COX, is induced in the presence of gastrointestinal mucosal inflammation. Although it has been reported that COX-2 derived prostaglandins (and probably $\mathrm{NO}$ derived from iNOS as well) are involved in gastric ulcer repair [22], they also contribute to perpetuation of gastrointestinal inflammation $[23,24]$. In the same way, induction of NOS may be associated with pathologic conditions. In the gastrointestinal tract, iNOS is activated in gastritis induced by Helicobacter pylori infection, inflammatory bowel diseases, and NSAID-induced ulcerogenesis $[10,25,26]$. The role played by and the effect of the excess of $\mathrm{NO}$ in these conditions is unclear. In some experiments, exogenous addition of $\mathrm{NO}$ protected against or reduced the severity of indomethacin-induced gastric damage in rats [16]. In contrast, when indomethacin was administered to iNOS knockout mice there was a reduction in the amount of gastric damage compared with that in wild-type animals [27]. This suggests that in this setting, NO generated from iNOS is involved in indomethacin-induced gastric lesions in the 
stomach. These apparently contradictory results illustrate the difficulty in elucidating the role played by NO in many functions, including NSAID-induced gastric damage. There are contradictory reports in which low levels of NO are seen to provide gastroprotection against NSAID-induced ulcerogenic damage [16,28], whereas higher concentrations lead to NSAID-induced damage $[29,30]$; this is similar to what has been suggested in various experimental models of colitis [10].

\section{Effects of nitric oxide donors on NSAID-induced damage to the gastrointestinal tract}

The mechanisms that underlie the ulcerogenic properties of NSAIDs are still being elucidated, but inhibition of mucosal prostaglandins is a key factor $[13,14,17]$. Various experimental models have shown that, in addition to the topical effect of NSAIDs and the COX dependent inhibition of prostaglandins, other important mechanisms are involved. Among these, we can highlight a decrease in mucosal blood flow and induction of leukocyte adherence within the gastrointestinal mucosal microcirculation as being two mechanisms of NSAIDs in which $\mathrm{NO}$ has a direct effect $[10,13,14,17]$. As commented on above, exogenous NO reduces gastric damage from NSAIDs in rats [16]. Other experiments also suggest that $\mathrm{NO}$ plays a role in reducing leukocyte adherence within the gastric microcirculation in the presence of indomethacin [10]. When rats were pretreated with the NO donor sildenafil, there was a significant reduction in indomethacin-induced gastric damage compared with untreated control animals, and this reduction was blocked by treatment with the NOS inhibitor L-NAME. Furthermore, leukocyte adhesion was significantly decreased in these animals, once again suggesting that NO-dependent mechanisms play a role in protection against NSAID-induced damage [31]. These examples help to define the gastroprotective effects of $\mathrm{NO}$ and suggest that $\mathrm{NO}$ donors could help to protect against NSAID-induced gastric damage.

The toxicity induced by NSAIDs in the intestine has some specific features. Unlike the stomach and the duodenal bulb, gastric acid is not present but the intestinal lumen contains bile and enterobacteria that potentiate the damage after the initial increase in gut permeability induced by NSAIDs [32]. The inflammatory response that follows these events is associated with induction of iNOS and increased NO production in the gut [33-35]. Although the precise effect of iNOS induction in these experimental settings remains unclear and excess NO may perpetuate damage, it has been shown that both $\mathrm{NO}$ derived from the different isoforms and prostaglandins are required to heal and protect intestinal lesions from NSAIDs [36,37].

Evidence generated from experiments and epidemiologic studies in humans suggest that exogenous $\mathrm{NO}$ has a protective effect on the gastrointestinal tract, specifically against NSAID-induced gastric damage. Figure 1 depicts
Figure 1

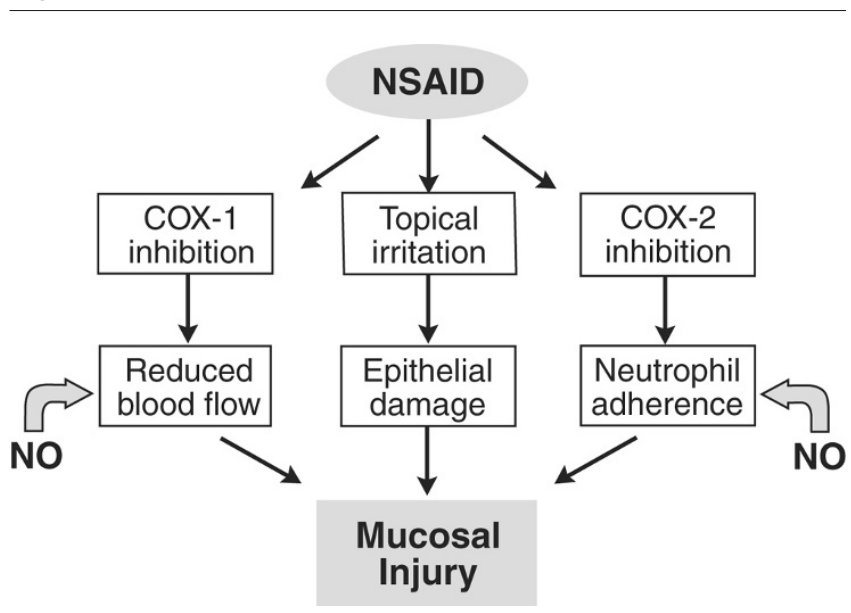

Potential targets for the gastroprotective effects of NO on NSAIDinduced gastric damage. COX, cyclo-oxygenase; NO, nitric oxide; NSAID, nonsteroidal anti-inflammatory drug. Data from Mannick and coworkers [26].

potential targets for the protective effects of $\mathrm{NO}$ against NSAID-associated pathogenesis [38].

\section{Dietary nitrate}

Experimental evidence has demonstrated that dietary nitrate can help protect against NSAID-induced gastric mucosal damage. Rats fed nitrate daily in their drinking water exhibited an increase in the thickness of gastric mucus and an upregulation of MUC6, which is a component of mucus in the gastric mucosa [39]. In addition, nitrate pretreatment reduced diclofenac-induced gastric lesions in a dose-dependent manner. Finally, inflammatory activity was decreased in nitrate-pretreated rats, as indicated by lower mucosal myeloperoxidase activity and a decrease in iNOS expression. In a similar study, Petersson and colleagues [40] demonstrated that rats pretreated with nitrate or given luminal nitrite had higher gastric mucosal blood flow than did untreated animals, again suggesting a gastroprotective role for NO. Because nitrite administered luminally in eNOS-null mice also resulted in increased gastric blood flow, the authors suggested that the gastroprotective effects of nitrate were likely the result of nonenzymatic NO production.

\section{Epidemiologic studies}

Epidemiologic studies have illustrated that treatment with nitrates can reduce the risk for upper gastrointestinal bleeding (UGIB) in patients taking NSAIDs. A case-control study was conducted to determine the risk for UGIB among patients taking low-dose aspirin, other NSAIDs, or nitrovasodilators (organic nitrates or nitroglycerin) was performed [41]. After adjustment for age, sex, and clinical risk factors (including a history of UGIB, a history of ulcer, and the presence of cardiovascular or cerebrovascular disease), the use of NSAIDs other than low-dose aspirin and use of low- 


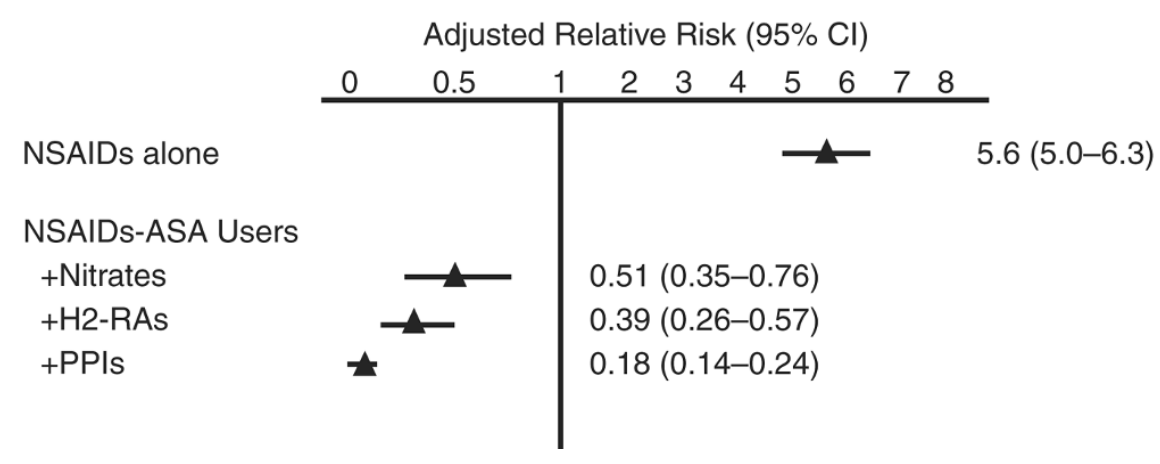

Risks for peptic ulcer bleeding. Presented are adjusted relative risks and 95\% confidence intervals for peptic ulcer bleeding associated with the use of nitrates and gastroprotective agents in the population using NSAIDs or aspirin. ASA, aspirin; $\mathrm{H} 2-\mathrm{RA}, \mathrm{H}_{2}$-receptor antagonist; $\mathrm{Cl}$, confidence interval; NSAID, nonsteroidal anti-inflammatory drug; PPI, proton pump inhibitor. Data from Lanas and colleagues [42].

dose aspirin were both associated with increased risk for UGIB. However, the use of nitrovasodilators or antisecretory therapy, including $\mathrm{H}_{2}$ receptor antagonists and proton pump inhibitors (PPIs), was associated with a decreased risk for UGIB, and this decrease was also observed when patients were receiving NSAID or aspirin therapy.

A more recent, larger case-control study [42], conducted after the withdrawal from the market of the COX-2 inhibitors rofecoxib and valdecoxib, confirmed these results. It once again illustrated that co-therapy with nitrates, PPIs, and $\mathrm{H}_{2}$ receptor antagonists all decreased the risk for UGIB associated with NSAID or aspirin use, although the protection conferred by nitrates was weaker than that by PPls (Figure 2) [42]. Intriguingly, a specific polymorphism in the eNOS gene that is associated with an increase in plasma $\mathrm{NO}$ concentrations was found to be associated with decreased risk for UGIB in lowdose aspirin users [43], again suggesting that additional NO helps to prevent damage or promote the healing of NSAIDinduced damage to the gastroduodenal mucosa.

\section{CINODs protect against NSAID-induced gastrointestinal damage}

As commented on above, NO has the capacity to block or compensate for the reduction in mucosal blood flow and can block the adherence of neutrophils to the vascular endothelium associated with NSAIDs in the gastrointestinal tract. Based on these properties, it was proposed that linkage of a NO-releasing moiety to an NSAID could reduce the gastrointestinal toxicity of these agents $[6,7,10,14]$. Seminal experiments in rats showed that NO-releasing derivatives of NSAIDs (including NO-flurbiprofen, NO-ketoprofen, NO-diclofenac, and NO-naproxen) can spare the gastrointestinal tract and be as effective as their parent drugs $[6,7,10,14,44,45]$.

NO-naproxen (naproxcinod) administered twice daily for 18 days resulted in significantly less gastric damage than did naproxen alone. In addition, naproxcinod also had improved analgesic and comparable anti-inflammatory activities when compared with naproxen [46]. Comparisons of flurbiprofen and NO-flurbiprofen showed that blood flow in the duodenum and mucus accumulation both decreased in the flurbiprofen treated animals compared with the NO-flurbiprofen groups [47]. Mucosal permeability also increased in the flurbiprofen but not NO-flurbiprofen treated animals. These findings suggest that the positive effects of NO-flurbiprofen may be due to its ability to maintain gastric blood flow, which may contribute to the reduced risk for NSAID-induced lesion. Finally, rats injected subcutaneously with indomethacin or NO-indomethacin (NCX 530) were analyzed after 24 hours [48]. Macroscopic observation revealed that indomethacin exposure led to gastrointestinal damage, primarily in the jejunum and ileum, along with increased inflammatory activity, as indicated by myeloperoxidase and iNOS activities. In addition, indomethacin injections decreased mucus and fluid secretions. In contrast, NCX-350 injections resulted in no gross gastric damage and decreased mucosal prostaglandin $\mathrm{E}_{2}$ content and inflammatory activity.

Some early clinical studies have also been conducted to test the effects of CINODs in humans. Naproxcinod was administered to 31 healthy individuals, and gastrointestinal injury was compared with that in participants who received naproxen or placebo [49]. Naproxcinod resulted in fewer gastroduodenal erosions in both the stomach and duodenum than did naproxen or placebo. In addition, naproxen alone increased intestinal permeability whereas naproxcinod and the placebo did not, suggesting that naproxcinod has an improved gastrointestinal profile compared with naproxen.

In another study [50], 970 patients were randomly assigned to receive naproxcinod $750 \mathrm{mg}$ twice daily, naproxen $500 \mathrm{mg}$ twice daily, or placebo twice daily in a double-blind study. Compared with baseline, significantly fewer ulcers and erosions developed in stomach and stomach/duodenum combined on naproxcinod than on naproxen. The incidence of 
Figure 3

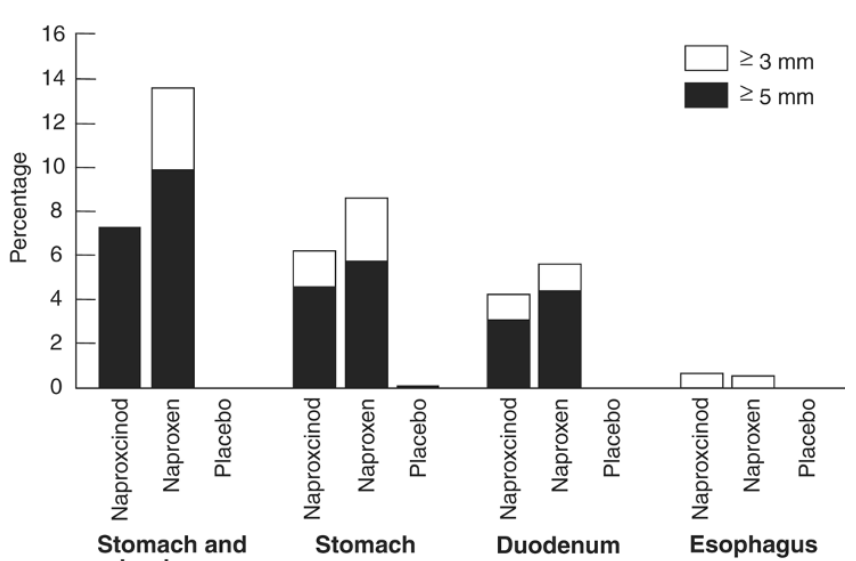

Incidence and location of ulcers in osteoarthritis patients treated with naproxcinod or naproxen. Data from Lohmander and colleagues [50].

ulcers (the primary end-point of the study) was $9.7 \%$ with naproxcinod versus $13.7 \%(P=0.07$, not significant $)$ with naproxen and $0 \%$ for placebo (Figure 3). The incidence of Lanza scores $>2$ was higher with naproxen $(43.7 \%)$ than with naproxcinod (32.2\%). Naproxcinod was as effective as naproxen at improving WOMAC (Western Ontario and McMaster Osteoarthritis Index) scores [50].

Similarly, NO-aspirin (NCX 4016) or aspirin was administered to healthy individuals, and the anti-inflammatory and antithrombotic effects as well as gastrointestinal tolerability were compared [51]. The study demonstrated that both NCX 4016 and aspirin had similar effects on platelet aggregation and inhibition of COX activity. However, NCX 4016 resulted in significantly less gastric damage than did aspirin alone, as indicated by endoscopy. In addition, unlike aspirin alone, NCX 4016 inhibited tissue factor expression on lipopolysaccharide-stimulated monocytes, suggesting that NCX 4016 has NO mediated activity in addition to COX inhibiting activity [51]. These studies confirm results from animal models and in vitro experiments, and suggest that CINODs could be new options in the treatment of OA pain that potentially have an improved gastrointestinal safety profile.

\section{Conclusion}

$\mathrm{NO}$ has been extensively studied and has well defined protective functions in the gastrointestinal tract, but when it is overproduced - as in the case of iNOS activation - the results can be pathogenic. Nevertheless, epidemiologic and laboratory experiments demonstrate that exogenous NO can either prevent or reduce the gastrointestinal toxicity associated with NSAIDs. Early research with CINODs supports the hypothesis that exogenous NO helps to protect against NSAID-induced gastric damage and/or promotes gastric healing. Although more research is needed, CINODs appear to be effective anti-inflammatory therapeutic agents with a potentially improved gastrointestinal safety profile compared with NSAIDs.

\section{Competing interests}

$\mathrm{AL}$ acts as an advisor to NicOx.

\section{Acknowledgments}

The author would like to thank Nina Leeds for editorial and referencing assistance with this manuscript.

This article is published as part of Arthritis Research \& Therapy Volume 10 Supplement 2, 2008: New insights in the role of nitric oxide in the management of osteoarthritis. The full contents of the supplement are available online at http://arthritis-research.com/supplements/10/S2.

Publication of the supplement has been supported by an unrestricted educational grant from $\mathrm{NicO}$.

\section{References}

1. Lanas A, Hunt R: Prevention of anti-inflammatory drug-induced gastrointestinal damage: Benefits and risks of therapeutic strategies. Ann Med 2006, 38:415-428.

2. Bresalier RS, Sandler RS, Quan H, Bolognese JA, Oxenius B, Horgan K, Lines C, Riddell R, Morton D, Lanas A, Konstam MA, Baron JA; Adenomatous Polyp Prevention on Vioxx (APPROVe) Trial Investigators: Cardiovascular events associated with rofecoxib in a colorectal adenoma chemoprevention trial. $N$ Engl J Med 2005, 352:1092-1102.

3. Laine L, Takeuchi K, Tarnowski A: Gastric muocsal defense and cytoprotection. Bench to Bedside. Gastroenterology 2008, 135: 41-60.

4. Hernandez-Diaz S, Rodriguez LA: Association between nonsteroidal anti-inflammatory drugs and upper gastrointestinal tract bleeding/perforation: an overview of epidemiologic studies published in the 1990s. Arch Intern Med 2000, 160: 2093-2099.

5. Wolfe MM, Lichtenstein DR, Singh G: Gastrointestinal toxicity of nonsteroidal antiinflammatory drugs. N Engl J Med 1999, 340:1888-1899.

6. Wallace JL, Reuter B, Cicala C, McKnight W, Grisham MB, Cirino G: Novel nonsteroidal anti-inflammatory drug derivatives with with markedly reduced ulcerogenic properties in the rat. Gastroenterology 1994, 107:173-179.

7. Wallace JL, Reuter B, Cicala C, McKnight W, Grisham M, Cirino G: A diclofenac derivative without ulcerogenic properties. Eur $J$ Pharmacol 1994, 257:249-255.

8. Moncada S, Higgs A: The L-arginine-nitric oxide pathway. $N$ Engl J Med 1993, 329:2002-2012.

9. Cho $\mathrm{CH}$ : Current roles of nitric oxide in gastrointestinal disorders. J Physiol Paris 2001, 95:253-256.

10. Wallace JL, Miller MJS: Nitric oxide in mucosal defense. A little goes a long-way. Gastroenterology 2000, 119:512-520.

11. Nathan $\mathrm{C}$ : Inducible nitric oxide synthase: what difference does it make? J Clin Invest 1997, 100:2417-2423.

12. Morin MJ, Karr SM, Faris RA, Gruppuso PA: Developmental variability in expression and regulation of inducible nitric oxide synthase in rat intestine. Am J Physiol Gastrointest Liver Physiol 2001, 281:G552-G559.

13. Wallace JL, Tigley AW: New insights into prostaglandins and mucosal defence. Aliment Pharmacol Ther 1995, 9:227-235.

14. Wallace JL: Non-steroidal anti-inflammatory drugs and gastroenteropathy: the second hundred years. Gastroenterology 1997, 112:1000-1016.

15. Pique JM, Whittle BJ, Esplugues JV: The vasodilator role of endogenous nitric oxide in the rat gastric microcirculation. Eur J Pharmacol 1989, 174:293-296.

16. Calatayud S, Sanz MJ, Canet A, Bello R, de Rojas FD, Esplugues JV: Mechanisms of gastroprotection by transdermal nitroglycerin in the rat. Br J Pharmacol 1999, 127:1111-1118.

17. Allen A, Flemstrom G, Garner A, Kivilaakso E: Gastroduodenal mucosal protection. Physiol Rev 1993, 73:823-857.

18. Brown JF, Keates AC, Hanson PJ, Whittle BJ: Nitric oxide generators and CGMP stimulate mucus secretion by rat gastric 
mucosal cells. Am J Physiol 1993, 265:G418-G422.

19. Brown JF, Hanson PJ, Whittle BJ: Nitric oxide donors increase mucus gel thickness in rat stomach. Eur J Pharmacol 1992, 223:103-104.

20. Kato S, Kitamura M, Korolkiewicz RP, Takeuchi K: Role of nitric oxide in regulation of gastric acid secretion in rats: effects of NO donors and NO synthase inhibitor. Br J Pharmacol 1998, 123:839-846.

21. Berg A, Redeen S, Grenegard M, Ericson AC, Sjostrand SE: Nitric oxide inhibits gastric acid secretion by increasing intraparietal cell levels of cGMP in isolated human gastric glands. Am J Physiol Gastrointest Liver Physiol 2005, 289:G1061-1066.

22. Schmassmann A, Zoidl G, Peskar BM, Waser B, SchmassmannSuhijar D, Gebbers JO, Reubi JC: Role of the different isoforms of cyclooxygenase and nitric oxide synthase during gastric ulcer healing in cyclooxygenase-1 and -2 knockout mice. Am J Physiol Gastrointest Liver Physiol 2006, 290:G747-G756.

23. Fiocchi C: Inflammatory bowel disease: etiology and pathogenesis. Gastroenterology 1998, 115:182-205.

24. Lauritsen K, Laursen LS, Bukhave K, Rask-Madsen J: Effects of topical 5-aminosalicylic acid and prednisolone on prostaglandin E2 and leukotriene B4 levels determined by equilibrium in vivo dialysis of rectum in relapsing ulcerative colitis. Gastroenterology 1986, 91:837-844.

25. Martin MJ, Jimenez MD, Motilva V: New issues about nitric oxide and its effects on the gastrointestinal tract. Curr Pharm Des 2001, 7:881-908.

26. Mannick EE, Bravo LE, Zarama G, Realpe JL, Zhang XJ, Ruiz B, Fontham ETH, Mera R, Miller MJS, Correa P: Inducible nitric oxide synthase, nitrotyrosine, and apoptosis in Helicobacter pylori gastritis: effects of antibiotics and antioxidants. Cancer Res 1996, 56:3238-3243.

27. Souza MH, Lemos HP, Oliveira RB, Cunha FQ: Gastric damage and granulocyte infiltration induced by indomethacin in tumour necrosis factor receptor 1 (TNF-R1) or inducible nitric oxide synthase (iNOS) deficient mice. Gut 2004, 53:791-796.

28. Elliott SN, McKnight W, Cirino G, Wallace JL: A nitric oxidereleasing nonsteroidal anti-inflammatory drug accelerates gastric ulcer healing in rats. Gastroenterology 1995, 109:524530.

29. Gurbuz V, Alican I, Berrak, Yegen C, Bozkurt A, Oktar B, Haklar $\mathrm{G}$, Yuksel $\mathrm{M}$, Kurtel $\mathrm{H}$ : Role of nitric oxide in indomethacininduced gastric mucosal dysfunction in the rat. Exp Physiol 1999, 84:319-332.

30. Lamarque $D$, Whittle BJ: Role of oxygen-derived metabolites in the rat gastric mucosal injury induced by nitric oxide donors. Eur J Pharmacol 1995, 277:187-194.

31. Santos CL, Souza MH, Gomes AS, Lemos HP, Santos AA, Cunha FQ, Wallace JL: Sildenafil prevents indomethacin-induced gastropathy in rats: role of leukocyte adherence and gastric blood flow. Br J Pharmacol 2005, 146:481-486.

32. Lanas A, Panes J, Pique JM: Clinical implications of COX-1 and/or COX-2 inhibition for the distal gastrointestinal tract. Curr Pharm Des 2003, 9:2253-2266.

33. Whittle BJ, Laszlo F, Evans SM, Moncada S: Induction of nitric oxide synthase and microvascular injury in the rat jejunum provoked by indomethacin. Br J Pharmacol 1995, 116:22862290.

34. Boughton-Smith NK, Evans SM, Laszlo F, Whittle BJ, Moncada S: The induction of nitric oxide synthase and intestinal vascular permeability by endotoxin in the rat. $\mathrm{Br}$ J Pharmacol 1993, 110:1189-1195

35. Takeuchi K, Yokota A, Tanaka A, Takahira Y: Factors involved in upregulation of inducible nitric oxide synthase in rat small intestine following administration of nonsteroidal anti-inflammatory drugs. Dig Dis Sci 2006, 51:1250-1259.

36. Takeuchi K, Hatazawa R, Tanigami M, Tanaka A, Ohno R, Yokota $A$ : Role of endogenous nitric oxide (NO) and NO synthases in healing of indomethacin-induced intestinal ulcers in rats. Life Sci 2007, 80:329-336.

37. Ohno R, Yokota A, Tanaka A, Takeuchi K: Induction of small intestinal damage in rats following combined treatment with cyclooxygenase-2 and nitric-oxide synthase inhibitors. J Pharmacol Exp Ther 2004, 310:821-827.

38. Wallace JL: Nitric oxide, aspirin-triggered lipoxins and NOaspirin in gastric protection. Inflamm Allergy Drug Targets 2006, 5:133-137.
39. Jansson EA, Petersson J, Reinders C, Sobko T, Bjorne H, Phillipson M, Weitzberg E, Holm L, Lundberg JO: Protection from nonsteroidal anti-inflammatory drug (NSAID)-induced gastric ulcers by dietary nitrate. Free Radic Biol Med 2007, 42:510518.

40. Petersson J, Phillipson M, Jansson EA, Patzak A, Lundberg JO, Holm L: Dietary nitrate increases gastric mucosal blood flow and mucosal defense. Am J Physiol Gastrointest Liver Physiol 2007, 292:G718-G724.

41. Lanas A, Bajador E, Serrano P, Fuentes J, Carreno S, Guardia J, Sanz M, Montoro M, Sainz R: Nitrovasodilators, low-dose aspirin, other nonsteroidal antiinflammatory drugs, and the risk of upper gastrointestinal bleeding. N Engl J Med 2000, 343:834-839.

42. Lanas A, Garcia-Rodríguez LA, Arroyo MT, Bujanda L, Gomollón F, Forné M, Aleman S, Nicolas D, Feu F, González-Pérez A, Borda A, Castro M, Poveda MJ, Arenas J; Investigators of the Asociación Española de Gastroenterología (AEG): Effect of antisecretory drugs and nitrates on the risk of ulcer bleeding associated with nonsteroidal anti-inflammatory drugs, antiplatelet agents, and anticoagulants. Am J Gastroentero/ 2007, 102:507-515.

43. Piazuelo E, Fuentes J, Garcfa-Gonzalez MA, Jimenez P, Lanas A: A case-control study of the association between polymorphisms of the endothelial nitric oxide synthase and glycoprotein IIla genes and upper gastrointestinal bleeding in users of low-dose aspirin. Clin Ther 2008, 30:121-130.

44. Reuter BK, Cirino G, Wallace JL: Markedly reduced intestinal toxicity of a diclofenac derivative. Life Sci 1994, 55:PL1-PL8.

45. Cuzzolin L, Conforti A, Adami A, Lussignoli S, Memestrina F, Del Soldato P, Benoni G: Anti-inflammatory potency and gastrointestinal toxicity of a new compound, NO-naproxen. Pharmacol Res 1995, 31:61-65.

46. Davies NM, Roseth AG, Appleyard CB, McKnight W, Del Soldato $P$, Calignano A, Cirino G, Wallace JL: NO-naproxen vs. naproxen: ulcerogenic, analgesic and anti-inflammatory effects. Aliment Pharmacol Ther 1997, 11:69-79.

47. Holm L, Phillipson M, Perry MA: NO-flurbiprofen maintains duodenal blood flow, enhances mucus secretion contributing to lower mucosal injury. Am J Physiol Gastrointest Liver Physiol 2002, 283:G1090-G1097.

48. Mizoguchi $H$, Hase $S$, Tanaka A, Takeuchi K: Lack of small intestinal ulcerogenecity of nitric oxide-releasing indomethacin, NCX-530, in rats. Aliment Pharmacol Ther 2001, 15:257-267.

49. Hawkey CJ, Jones Jl, Atherton CT, Skelly MM, Bebb JR, Fagerholm U, Jonzon B, Karlsson P, Bjarnason IT: Gastrointestinal safety of AZD3582, a cyclooxygenase inhibiting nitric oxide donator: proof of concept study in humans. Gut 2003, 52: 1537-1542.

50. Lohmander LS, McKeith D, Svensson O, Malmenas M, Bolin L, Kalla A, Genti G, Szechinski J, Ramos-Remus C: A randomised, placebo controlled, comparative trial of the gastrointestinal safety and efficacy of AZD 3582 versus naproxen in osteoarthritis. Ann Rheum Dis 2005, 64:449-456.

51. Fiorucci $S$, Mencarelli A, Meneguzzi A, Lechi A, Renga B, del Soldato $P$, Morelli A, Minuz P: Co-administration of nitric oxideaspirin (NCX-4016) and aspirin prevents platelet and monocyte activation and protects against gastric damage induced by aspirin in humans. J Am Coll Cardiol 2004, 44:635-641. 